\title{
Stefano Brugnolo
}

\section{Sur les avantages et les désavantages des littératures périphériques}

\section{ON THE AdVANTAGES AND DisadVANTAGES OF PERIPHeRAL Literature}

Abstract: Taking as a starting point the volume Romanian Literature as World Literature, the essay investigates the relationships between the so-called major and the so-called minor literature. It also challenges all those approaches which claim that in the era of globalization and the Internet, the literary scene is a flat space where continental, national, and regional conditions of hegemony or subalternity are no longer present. The essay further questions the paradox according to which critical operations aimed at contesting certain cultural hegemonies, and primarily the United States, have better reception when promoted by institutions belonging to hegemonic cultures than when they are promoted by institutions of peripheral countries.

Keywords: Minor Literature; Peripheral Literature; Major Culture; World-system; World Literature; Romanian Literature.

\section{Stefano BRUgnolo}

Université de Pise, Italia

brugnolos@gmail.com

DOI: 10.24193/cechinox.2020.39.22
Tous ferons souvent référence, tout au 1 long de ce travail, à Romanian Literature as World Literature ${ }^{1}$, et essayerons de mettre en évidence les idées et les concepts fondamentaux que peut en retirer quiconque enseigne la littérature comparée et la théorie de la littérature. Le premier point qu'il convient de noter est que tous les spécialistes qui ont apporté leur contribution au volume contestent naturellement le paradigme historico-national. Autrement dit, ils remettent en question l'idée qu'une littérature écrite par des auteurs qui appartiennent à telle ou telle nation et qui écrivent dans telle ou telle langue puisse être analysée et comprise exclusivement comme le produit de la culture nationale en question.

Cela se fait jour dès l'introduction, qui est une sorte de Manifeste signé par Christian Moraru et Andrei Terian², où l'on trouve des expressions auxquelles nous souscrivons largement, comme celle-ci, tirée d'un travail de Nikos Papastergiadis : " l'art ne peut pas être compris comme une activité sociale qui atteint les objectifs déclarés d'un agenda national », et « la place spécifique de l'art se situe aujourd'hui de plus en plus au sein de réseaux qui sont à la fois au-dessus et au-dessous de la portée de l'État-nation $»^{3}$. 
Tout aussi valable est l'affirmation d'un autre chercheur, Alex Goldiș, qui cite Wai Chee Dimock, lequel soutient que « la littérature d'un pays est un 'enchevêtrement de relations' qui dépasse de loin l'espace national ou, plus exactement, le territoire qui se trouve sous l'administration de ce pays »; en effet, « il n'y a rien de naturel ou d'évident concernant le concept d'histoire littéraire entendu comme construction territorialement organique, en vertu duquel les frontières littéraires et nationales sont les mêmes " ${ }^{4}$. Tout à fait, mais ajoutons que cela ne vaut pas qu' « aujourd'hui » : la littérature, quand elle est grande, ne coïncide jamais avec les frontières des États et des nations, et ne constitue jamais non plus une émanation spontanée d'un ethos national supposé. Et la chose est évidemment plus vraie encore depuis le XVIII ${ }^{\mathrm{e}}$ siècle, c'està-dire lorsque le monde devient un unique système-monde, pour faire nôtre la formule de l'historien et du sociologue Immanuel Wallerstein, qui a souvent été cité ici ${ }^{5}$.

Nous pensons que cette perspective, bien qu'elle continue à faire face à une forte résistance de la part du système scolaire et universitaire et des institutions politiques et culturelles nationales, est tout à fait raisonnable, et que ce livre va, par conséquent, dans la bonne direction. Pour donner quelques exemples italiens, nous nous demandons si l'on peut réellement comprendre Manzoni sans le mettre en relation avec le romantisme et le siècle des Lumières européens ; sans le mettre en relation avec la Révolution française de 1789 et la naissance de la civilisation bourgeoise ; sans faire de parallèles avec Scott, Stendhal ou Balzac. C'est ainsi que toutes les opérations qui, comme celle-ci, nous permettent de lire les classiques d'un pays en les confrontant aux classiques d'un autre pays et, plus encore, en les situant dans un contexte international, sont les bienvenues.

Mais à deux conditions. La première est de faire preuve de la même méfiance à l'endroit de tous les autres particularismes et essentialismes, aussi bien de nature sexuelle dans le cas du féminisme et de l'homosexualité, que ceux qui se fondent sur des oppositions de type ethnique et politique, comme par excellence dans le cas des Noirs opprimés dans leur propre patrie ou dans celle des peuples blancs. Sans parler d'autres approches qui se fondent sur des bases territoriales ou régionalistes. Quel sens cela a-t-il de parler de littérature (de philosophie, de musique ou de peinture) féminine, homosexuelle, noire, juive ou sicilienne, comme s'il s'agissait de provinces à part ? $\mathrm{Si}$ l'on veut vraiment promouvoir l'idée de la littérature comme réalité internationale (ou transnationale comme préfèrent le dire nos auteurs), alors il convient d'avoir le courage de liquider tous les particularismes.

La seconde condition est qu'au nom d'une vision mondiale ou planétaire de la littérature, on n'en vienne pas à nier l'existence des spécificités culturelles, historiques, linguistiques et donc inévitablement territoriales et géopolitiques. Dans ce livre, les auteurs prennent souvent, à juste titre, leurs distances vis-à-vis du paradigme herdérien du peuple-nation, de même qu'ils ont recours à Benedict Anderson ${ }^{6}$ pour déclarer que les nations sont des « communautés imaginées », et non des essences ou des choses qui sont données de fait.

Sur le principe, nous sommes d'accord, mais cela ne devrait pas ensuite conduire, pour le plaisir de déconstruire les concepts et au nom d'un parti pris anti-essentialiste et anti-identitaire, auquel il est fait souvent 
référence dans le livre, à nier la réalité et l'importance de ces entités imaginaires que sont les nations. L'Italie et la Roumanie n'existent pas à l'égal d'une pierre ou d'une plante, soit, mais la Roumanie et l'Italie ne sont pas que des noms ou des signifiants ; ce sont des réalités inventées, construites, mais des réalités quand même, avec lesquelles il faut composer, notamment en tant que chercheurs en littérature.

Une chose est donc de s'abstenir de réifier ces appartenances, une autre de les liquider comme si elles n'existaient pas du tout ou étaient tout à fait insignifiantes. Il faut préciser, d'emblée, que de telles identités se définissent surtout sur une base relationnelle ; par exemple, ce n'est que par opposition aux Perses que les Grecs ont pu se penser comme Grecs. Et cela continue d'être vrai : on est roumain ou italien surtout par rapport à d'autres identités qui sont tour à tour admirées ou rejetées. En d'autres termes, en tant que chercheurs en littérature c'est le jeu entre la dimension nationale et la dimension internationale qui devrait nous intéresser, et non l'annulation de l'une à l'intérieur de l'autre.

Une littérature mondiale, une Weltliteratur, peut constituer un idéal même éthique, et l'on trouve souvent dans ce livre des traces de cette inspiration; que l'on voie par exemple cet extrait, où il est question d'un nouvel espace culturel et littéraire : "cet espace n'est pas organisé selon une hiérarchie de centres et de marges. Il ne ressemble pas du tout non plus aux territoires de ces pays qui érigent des murs et des barrières pour empêcher les immigrants, les réfugiés et d'autres indésirables d'entrer $»^{7}$.

Il est évident que les auteurs qui prennent parti pour une littérature sans frontières, sopposent aux nouvelles tendances nationalistes et racistes de plus en plus répandues, celles qui entendent justement ériger des murs. Partant de là, ils soutiennent que si l'on adoptait le paradigme transnational, on se libérerait du préjugé selon lequel la littérature des périphéries serait une " reproduction du centre esthétiquement inférieure - d'où les complexes apparentés de 'marginalité', 'provincialité', 'arriération', 'dérivation', 'retard' et 'infériorité' ${ }^{8}$.

Or, il va de soi que toute qualification d'infériorité correspond à un préjudice, mais peut-on dire la même chose d'autres réalités que sont le retard de développement et le caractère périphérique ? On lit ailleurs que durant deux siècles, "la scalarité culturelle et épistémologique a été l'État-nation, à travers lequel l'humain, dans son expression culturelle, et le monde humain, sont devenus intelligibles pour au moins 200 ans ", et, qu'à présent " il nous faut considérer la planète entière comme une unité d'analyse " ${ }^{9}$. Nous répétons : pareilles prises de position sont moralement et politiquement acceptables, mais il faut se demander si d'un point de vue scientifique, il est vraiment possible de considérer la planète (!) comme une « unité d'analyse " ? Si la nation risque dêtre un point de référence trop étroit, le monde risque, lui, d'être trop vague et générique.

Le pathos du discours est donc compréhensible, certes, mais ne devrait pas nous pousser à être irréalistes et à oublier que les logiques étatiques et nationales tiennent encore bon et influent sur tous les phénomènes culturels et littéraires. Cela ne devrait pas nous porter à embrasser, de manière acritique, la rhétorique de la mondialité comme en témoignent certaines des expressions utilisées : 
Quelle littérature n'est pas nomade ? Quelle littérature n'est pas 'immigrée' ? Quelle littérature ne dépend pas - et parfois même ne 'naît' pas d'une traduction? [...]. Et quelle production littéraire n'est pas transculturelle et transterritoriale ? $^{10}$.

Aucune nation et aucun peuple ne se situent évidemment hors d'un tel flux, mais reste cependant à voir les façons dont les différents sujets participent à ce grand jeu d'échanges et d'influences. Certaines affirmations attestent un esprit militant qui, parce qu'il aspire à l'abolition des différences et des inégalités, imagine que la chose est déjà effective, et que tout n'est qu'hybridisme, nomadisme, échange, flux, sans limites, sans murs et sans hiérarchies. On se demande en effet si les auteurs pensent réellement qu'il n'y a pas d'écarts de vitesse en termes de développement économique, scientifique, culturel et finalement littéraire des différentes parties du monde ? Rappeler que ces écarts existent ne signifie pas que l'on ait affaire à des littératures inférieures et supérieures. Cela signifie se souvenir que s'il est vrai que le champ littéraire comme le champ culturel sont constitués de flux, dans ce réseau d'échanges, la circulation a rarement été bilatérale et paritaire.

Et il n'est pas dit, de toute façon, que le besoin de nation corresponde seulement à un habitus de fermeture; les luttes de certaines populations devraient nous en convaincre. C'est pourquoi un certain parti pris anti-national peut aussi faire figure de contre-idéal, de dystopie, si, dans ce contexte, aucune place n'est faite aux différences, qui sont assurément de nature variée, mais linguistiques et historico-culturelles au premier chef.
La question paradoxale que se pose Montesquieu : "Comment peut-on être persan?" permettait d'apporter une réponse à l'autre question : "Comment peut-on être européen ? ». Autrement signifié, nous avons besoin de lire des livres roumains pour pouvoir répondre à la question «Comment peut-on être roumain ?", mais aussi pour répondre à la question " comment peut-on être italien ? ", et inversement. Mais il est vrai aussi que ce n'est souvent que par le regard extérieur qu'un peuple s'identifie : l'italianité moderne, par exemple, est à bien des égards une invention de Goethe, de Byron, de Stendhal et d'autres encore, plus qu'un produit de l'imagination autochtone.

Ce n'est qu'à travers ces perspectives croisées et systématiques que nous pouvons comprendre mieux qui nous sommes, et en nous reflétant dans l'autre nous comprendre nous-mêmes : c'est sur cette base que s'appuie l'approche comparatiste des études littéraires. Mais ces reflets réciproques sont à chaque fois différents et spécifiques. Prétendre adopter comme unité de mesure la planète risque de rendre nos comparaisons vagues et peu efficaces : comment saisir l'originalité du roman russe (ou japonais ou latino-américain) et sa grande contribution au roman comme genre planétaire si l'on néglige totalement le contexte géographique, culturel, linguistique dans lequel il est né ? En Europe se sont produits énormément de « jeux » similaires, et certains ont concerné la Roumanie, surtout en relation avec la France (mais aussi avec l'Allemagne). L'Europe, ne l'oublions pas, a également été cela, en effet : un extraordinaire enchevêtrement de différences, et tout parti pris anti-nationaliste juste ne devrait pas nous faire oublier que la diversité culturelle et donc littéraire est une valeur. 
Franco Moretti est souvent cité dans l'ouvrage, mais l'une de ses idées, qui me semble être une idée forte de sa conception de world-literature ${ }^{11}$, est souvent contestée, à savoir que s'il est vrai qu'il existe un espace littéraire mondial parallèle à celui économique, il est vrai aussi que ces deux espaces ne sont pas homogènes, plats, horizontaux, et ce, parce qu'aussi bien le développement de l'économie que celui de la culture et de la littérature sont inégaux. Cela revient à dire que les deux sont traversés par de grandes contradictions et des différences surtout économiques, la principale étant celle qui distingue le centre ou les centres des périphéries.

Moraru, Terian et les autres auteurs, à l'inverse, quoiqu'ils dialoguent avec Moretti, privilégient un paradigme moins « dramatique ", et peut-être aussi plus consolateur : ils parlent souvent de nœuds et d'intersections entre cultures et littératures (ils utilisent continuellement les adjectifs nodal et intersectional, ou les substantifs intersection et nodality), et mettent donc l'accent sur la dimension polyphonique et multilatérale des cultures et des littératures contre l'opposition binaire et monodirectionnelle entre centre et périphérie. Ils soutiennent en effet qu'il faut « traiter la littérature nationale comme 'un point nodal particulier' (Beebee) dans un ensemble plus vaste ", et s'opposent à une " vision plutôt simpliste des mécanismes culturels qui régulent les centres et les marges $»^{12}$.

Ils ne nient donc pas que l'on peut parler de littératures nationales, mais celles-ci devraient être comprises comme des nœuds, des points d'intersection vers lesquels convergent des flux dans toutes les directions : dans cette perspective, la nation ne serait pas autre chose que " échantillon coloré et vibrant de tout ce qui se retrouve, se touche, se classifie, se mélange, passe dans la nation et à travers elle $»^{13}$. Mais encore une fois il semble que ce soit l'instance éthique plus que scientifique qui prévale : il est vrai qu'une nation peut être comprise comme un point d'articulation et un carrefour de plusieurs courants, mais il n'est pas vrai que les flux s'effectuent selon des modalités désordonnées et stochastiques comme ces auteurs pensent le croire dès lors qu'ils souscrivent à une affirmation de Wai Chee Dimock : « la littérature pour la planète est 'un ensemble décentré de vibrations, chaotique et tangentiel', un champ d'énergie, un flux $»^{14}$.

Pour parvenir à démontrer qu'il n'existe pas d'excellences, de même qu'il n'existe pas d'infériorités, certains chercheurs optent pour un paradigme de type intertextuel, selon lequel la littérature serait un monde autoréférencé, une Bibliothèque de Babel borgésienne, où chaque livre renvoie infiniment à un autre livre. Il s'agit d'une vision qui fait aujourd'hui son chemin chez beaucoup de théoriciens de la World Literature, mais en grande partie erronée, nous semble-t-il, parce que nous croyons qu'une république mondiale des lettres (pour le dire à la façon de Pascale Casanova $)^{15}$ ne peut se concevoir que si on l'entend comme internement divisée et dramatiquement inégale.

Pour mieux en rendre compte, peutêtre aurait-il été utile d'en appeler à Gramsci et à son concept d'hégémonie en dépassant le contexte national auquel il le cantonne pour renvoyer aux hégémonies culturelles et idéologiques, avant même d'être littéraires, qui s'exercent à 
l'échelle internationale. Aucune perspective polyphonique et poly-systémique ne peut, en effet, selon nous, effacer complètement la réalité d'un monde où le pouvoir littéraire et, en général, culturel, comme le pouvoir économique, tendent à l'accumulation et à la concentration, et non pas à la dissémination.

Il ne suffit donc pas de dire, comme le fait Teodora Dumitru, que chaque littérature « existe à l'intérieur d'un flux constant de traductions de textes qui viennent d'ailleurs et à destination du marché mondial ${ }^{16}$, il convient aussi de préciser mieux comment, combien, pourquoi et ce que l'on traduit d'une langue à l'autre. Aucune perspective intertextuelle ne pourra nier par exemple le fait que la primauté culturelle qui fut longtemps exercée par la France et par Paris est aujourd'hui exercée par les États-Unis, ses prestigieuses universités, ses grandes maisons d'édition, ses revues et ses fondations.

Penchons-nous sur ces théories et sur tous ces théoriciens qui prônent justement une approche de la culture et de la société de type démocratique, polycentrique, polyphonique, ouverte, contre-hégémonique, antisexiste, anti-classiste... eh bien, ces théoriciens ont une chance de se faire écouter et entendre si, et seulement si, ils parlent depuis ces centres. Cela nous semble être un paradoxe sur lequel il vaut la peine de s'interroger : on peut intervenir contre les inégalités du système culturel mondial, et espérer susciter des réactions chez le plus grand nombre, à condition de parler depuis une position privilégiée à l'intérieur du système. Romanian Literature as World Literature, de la même manière, peut être lu, commenté, discuté, d'abord parce qu'il est édité par une maison d'édition comme Bloombsbury. Parler au monde de la littérature roumaine, italienne ou finlandaise comme littérature « du monde ", mais en le faisant depuis ces mêmes cultures et ces mêmes langues n'aurait aucun effet significatif sur le débat culturel international ${ }^{17}$.

Ceci étant dit, il faut tout de suite rappeler que l'hégémonie telle qu'elle est définie par Gramsci est un concept qui ne fonctionne pas que dans une direction ${ }^{18}$; au contraire, il estime que l'hégémonie d'une classe comme d'une nation est de toute façon soumise à négociation, à conflit, mais toujours, précisément, dans le cadre de rapports de force déterminés ; ce qui ne peut être compris que si l'on adopte un modèle de type antagonistique plutôt qu'un modèle « nodal » ou « intersectionnel » dans lequel tout est flux et échange plus que lutte pour une quelconque place prédominante.

Les apories du modèle intersectionnel se font jour avec plus de clarté si l'on se tourne vers les arts dits commerciaux. Pense-t-on vraiment que dans le monde de la musique rock ou pop, par exemple, il n'existe ni centres ni périphéries, et qu'il n'y a que des intersections, des influences et des échanges à jeu égal entre artistes anglais et américains, d'une part, et artistes roumains, italiens, moldaves, etc., d'autre part ? Et on peut dire la même chose, naturellement, du cinéma comme de genres plus récents, par exemple des séries télévisées. Il n’y a pas à se plaindre de façon victimiste de cette situation, qui, sous certains aspects, va de soi, et encore moins à assumer des positions défensives et nationalistes, régionalistes ou anti-américaines, mais il vaut la peine d'être réaliste et de cesser de dire, quoi qu'il en soit, que nous vivons dans un monde d'échanges culturels et artistiques libres entre partenaires qui se trouvent sur un pied d'égalité parfaite. 
L'une des principales limites de cette approche transnationale de la littérature est la sous-évaluation totale du lieu, de la position depuis laquelle parle un auteur, et l'emphase qui est mise sur les processus de déterritorialisation (un autre mot récurrent dans le volume dont nous nous occupons). De notre point de vue, quiconque affirme que le monde est un espace unique et indifférencié se trompe, de même que celui qui met l'accent sur les diversités nationales, ethniques jusqu'à les réifier ; seule une approche qui tout en reconnaissant qu'il existe un unique système-monde prenne en considération des différences et des inégalités de développement des sociétés, des cultures et des littératures peut rendre compte des phénoménologies littéraires modernes, et non pas une approche intertextuelle qui abolit la dimension historique et son caractère dramatique.

Nous disons tout cela parce que nous voudrions soutenir la thèse que la condition dite périphérique peut aussi être un avantage cognitif, ce que nous avons appelé, ailleurs, le privilège du retard. L'idée serait que les écrivains périphériques peuvent reconsidérer de façon originale et dans un second temps (après coup) tout ce qui dans les métropoles et dans les centres du système-monde est considéré comme allant de soi et naturel. Il ne s'agit donc pas de nier qu'il existe des primautés et des hiérarchies, mais il s'agit de dire que l'auteur périphérique peut transformer en une opportunité poétique une condition de retard apparent. Voici comment nous avons défini cette situation, surtout en relation avec Dostoïvski et les auteurs russes du $\mathrm{XX}^{\mathrm{e}}$ siècle :

Nous pouvons parler de réponses différées ou retardées de la Périphérie comme réaction aux processus de modernisation mis en œuvre par le Centre. C'est presque comme si les écrivains périphériques avaient, au moins d'un point de vue théorique, le privilège de reconsidérer et de redéfinir après coup ces processus qui se sont stabilisés ailleurs, qui sont devenus une sorte de seconde nature. J'ai appelé ce phénomène le privilège de l'arriération ${ }^{19}$.

Il est donc vrai que les écrivains périphériques dépendent de ceux qui sont centraux, mais il n'est pas vrai qu'ils se contentent de les imiter; ils leur répondent plutôt créativement et, ce faisant, ils modifient radicalement thèmes, procédés et styles. Il nous semble que c'est ce mouvement d'action-réaction entre différentes zones et littératures du monde qui devrait être davantage étudié, d'autant plus que c'est souvent dans les périphéries du monde ou dans les interstices de ce système, en tout cas, que l'on peut le mieux en saisir les contradictions.

Aujourd'hui, à l'heure d'internet, peut-être n'existe-t-il plus de zones développées, moins développées ou sous-développées ? C'est en effet avec cet esprit que beaucoup pensent la mondialisation, aussi bien à droite qu'à gauche de l'échiquier politique.

Il est vrai, certes, qu'une énorme masse d'individus (mais pas tous quoi qu'il en soit) sont online, mais cela suffit-il à nous rendre culturellement homogènes et paritaires ? La mondialisation des marchés a-t-elle été de pair avec la naissance d'une citizenship mondial ? La prédominance de l'anglais a-t-elle aboli les autres langues parlées ou plutôt, les a-t-elle marginalisées ? Pourquoi 
donc ne pas penser que la contemporanéité $\mathrm{du}$ non-contemporain dont parlait le philosophe Ernest Bloch continue de traverser et de diviser nations et continents, et qu'elle « coupe » même actuellement les vies des individus singuliers dans n'importe quel lieu ? Des hommes et des femmes peuvent être hyperconnectés et faire un usage quotidien des médias les plus à la pointe et partager, dans le même temps, des mythes, des valeurs, des croyances, des peurs irrationnelles et archaïques.

Il en résulte que faire l'hypothèse, dans ce contexte, que n'existent que les échanges et les flux d'égal à égal entre écrivains, penseurs, artistes, intellectuels, cela signifie adopter une perspective idéaliste et célébrative des processus d'homologation de la mondialisation. Au contraire, les études comparatistes gagneraient à assumer qu'il existe une dialectique entre le local, le traditionnel, le territorial d'un côté, et le global, l'hypermoderne, le transnational de l'autre. En d'autres termes, il est vrai que le roman moderne est toujours orienté vers le monde et qu'il présuppose depuis longtemps un lecteur international, mais il l'est toujours à partir d'une réalité, d'une langue, d'une culture et de contextes spécifiques.

Nous croyons, en revanche, que la conviction exaltante selon laquelle il n'existe plus de frontières, d'appartenances, d'identités, de limitations nationales, mais que seuls comptent les phénomènes d'hybridation et de nomadisme culturel et littéraire, a vraiment à voir avec l'idéologie d'internet - compris mythiquement comme espace accessible et praticable par tous -, a à voir donc avec les illusions et les euphories que ce dernier a suscitées chez beaucoup de ses utilisateurs et propagandistes. De même que l'idée que le virtuel, la simulation ont remplacé le réel, le territorial, le naturel, a à voir avec cette même idéologie. Devant ces illusions, qui ne tiennent aucun compte des contre-réactions puissantes de type identitaire que la mondialisation produit, il faut insister sur le fait que même à l'heure d'internet, de la téléphonie mobile, le monde reste divisé et inégal.

Nous sommes au fond face à la vieille aporie en vertu de laquelle à une égalité sur le plan formel (sur le papier, nous sommes tous des citoyens libres de la grande République du web) ne correspond pas une égalité substantielle, ne serait-ce que parce qu'il est faux d'assumer que le virtuel s'est substitué au réel. En définitive, on pourrait dire que toute l'emphase qu'une certaine pensée postmoderne dans ses acceptions diverses met sur l'immatérialité, sur la vitesse, sur la multilatéralité des flux d'informations, sur les connaissances, sur les énergies, tend à nier de façon idéaliste certaines réalités de longue, de très longue durée : hier comme aujourd'hui nous naissons et nous existons dans certains espaces-temps bien déterminés, nous continuons à avoir des origines, des parents et des familles, à dépendre de notre enfance, à parler des langues spécifiques, à appartenir à une communauté, à avoir des corps fragiles et irremplaçables. Tout cela nous marque profondément, et ce nest pas un hasard si la littérature, et surtout le roman, nous parle si souvent de ce qui persiste d'ancien, de corporel, d'animal dans l'homo postmodernus. Aucune rhétorique multiculturaliste, posthumaine et liquide peut éliminer, en effet, ces lourdes réalités de base.

Et les migrants eux-mêmes le savent mieux que quiconque : ils sont souvent mentionnés par les théoriciens de la transnationalité sans limites et sans confins, mais ce sont eux précisément qui doivent 
en réalité faire face, au prix d'efforts et d'une souffrance physique et psychique, à des expériences de déracinement et de réadaptation, qui sont sous-évaluées au nom des valeurs exaltantes de l'hybridisme, du métissage, du nomadisme (comme si être nomade était toujours un choix, et non une nécessité pénible).

La migration peut aussi être une grande occasion de rebrassage des identités, et par conséquent de liberté et d'émancipation à l'endroit du passé et des appartenances traditionnelles, et les études littéraires doivent aussi tenir compte de ces nouvelles réalités, mais cela ne doit pas nous pousser à croire que de tels processus adviennent dans un espace-temps comparable à celui d'internet : ceux-ci impliquent un prix à payer, des conflits, des déséquilibres et des organisations nouvelles. Et ceux qui, réfugiés et migrants, écrivent sur leur expérience ne le font pas, de toute façon, en se plaçant dans un quelconque hyperespace transnational, mais plutôt dans une nouvelle patrie et une nouvelle langue, acquises souvent à grand-peine.

Dire de ces auteurs qu'ils sont tout à fait étrangers à toute dynamique d'inclusion et d'exclusion est un choix qui ne nous aide pas à mieux les comprendre. Il convient de se demander chaque fois : d'où provient cet auteur? Quelle est sa langue maternelle et la langue qu'il a apprise ? De quelle réalité parle-t-il, celle dont il est issu ou celle dans laquelle il s'est établi ? Des situations de cette nature ont toujours existé au fond, mais nous dirions qu'aujourd'hui, plus qu'hier, c'est cette dialectique entre appartenances et origines d'un côté, et désappartenances, différences et distances de l'autre, qui caractérise la plupart de la grande littérature contemporaine (que l'on pense ici à l'important filon anglo-indien, de Naipaul à Rushdie, en passant par Kureishi, et tant d'autres). Grâce à cette double perspective, interne et externe sur les choses, ces auteurs parviennent à transcender les réalités nationales dont ils parlent également avec force vivacité : l'Inde de Rushdie n'est pas un lieu semblable à tant d'autres.

Et cela nous donne le droit de dire dans quel sens on peut et on doit encore parler de réalités nationales quand on s'occupe de littérature, c'est-à-dire d'un discours qui, nous l'avons vu, transcende toujours ces réalités (comme le font en définitive la musique et les autres arts). Ce que Rushdie a fait avec «son » Inde, c'est ce qu'ont fait tous les grands romans modernes depuis le $\mathrm{XX}^{\mathrm{e}}$ siècle, à savoir transfigurer une situation fortement caractérisée en termes d'ambiance et de culture pour la transformer en une situation qui concerne potentiellement tout le monde ; transformer un lieu géographique très bien identifié en un lieu de l'esprit ouvert à tous.

Dit en d'autres termes encore, ces œuvres traitent d'une certaine zone, région, nation comme un sous-ensemble d'ensembles plus généraux. C'est ainsi que le Congo de Conrad, la Dublin de Joyce, le Sud des États-Unis de Faulkner, le Sertão de Guimaroes Rosa, la Prague de Hrabal ne sont pas tant ou seulement des zones réelles du monde représentées par ces auteurs de façon éclatante, mais aussi des zones du monde transfigurées par leur imagination et qui grâce à des processus d'expansion du sens illustrent donc des conditions et des situations bien plus générales. C'est ainsi que Manea et Cărtărescu, quand ils parlent de Bucarest, font allusion à deux réalités imaginaires différentes bien qu'ils renvoient tous les deux à la même ville. 
En ce sens nous nous permettons d'employer un mot aujourd'hui très suspect et de dire que ces auteurs ont tâché de rendre universelles des nations, des villes, des régions absolument singulières et spécifiques. Le mot s'avère suspect parce qu'on l'associe à un abus commis par les classes ou les puissances dominantes, pour imposer leurs propres valeurs comme universelles. Mais dans ce cas, dire que la Dublin provinciale et claustrophobique de Joyce ou le Sud défait et décadent de Faulkner sont des lieux universels n'implique aucun abus ou prépotence idéologiques, c'est même le contraire : nous tenons pour universels ces livres qui suscitent en nous des identifications spontanées, qui nous font sentir que ces mondes - si souvent périphériques, arriérés, lointains - sont nôtres, appartiennent à tous.

Voilà pourquoi des opérations comme celle que tente Romanian Literature as World Literature sont précieuses : ce qui nous intéresse vraiment, ce n'est pas tant ce qu'a été et ce qu'est la littérature roumaine en tant que littérature roumaine, mais ce que cette littérature peut dire au monde, précisément à partir de sa spécificité et de son unicité. Ce qui nous intéresse est ce que nous pouvons comprendre du monde en regardant à l'intérieur du miroir d'une littérature nationale soi-disant mineure. S'il y a donc une invitation que nous devons accepter, c'est celle que nous tirons de cet extrait : " l'un des principes fondamentaux des essais de Romanian Literature as World Literature est que [...] cette littérature passe par l'affirmation de son appartenance planétaire, de son être dans le monde, pour lui et de lui $»^{20}$, et il va de soi que cela peut et doit valoir pour toutes les autres littératures nationales. Mais justement, affirmons-le : la seule manière d'être planétaire est de rendre universels les réalités locales, le ici et maintenant dans lesquels chacun de nous vit.

Pour résumer, nous avons soutenu que la littérature est un champ ouvert à tous, mais nous avons aussi dit qu'il existe des littératures majeures et mineures, c'est-à-dire des littératures qui ont à certaines époques un pouvoir d'irradiation plus ou moins grand. Et pourtant dans certaines occasions, il peut arriver que des auteurs originaires des zones périphériques sachent parler de façon si efficace aux lecteurs centraux, et en définitive à tous, qu'ils deviennent canoniques, qu'ils deviennent des classiques. Certains des auteurs que nous avons nommés précédemment appartiennent justement à ces parties du monde. Et c'est précisément ce qui est arrivé à certains écrivains roumains.

Nous pensons par exemple à la façon dont un certain type d'humour dit de l'absurde s'est évidemment imposé dans le monde à travers l'œuvre de Ionescu (ou Ionesco). Évidemment Ionescu a écrit le gros de son œuvre en français, mais dans son cas aussi, on peut dire que ce qui a fait de lui un auteur original, c'est aussi et précisément sa condition d'écrivain exilé, à cheval entre plusieurs cultures. En effet, il y a beaucoup d'anticipations du comique de l'absurde avant lui, mais c'est Ionescu qui réussit à inventer ce type de discours qui a ensuite influencé et continue d'influencer tant d'écrivains et d'artistes. Or, nul doute, selon nous, que Ionescu est devenu l'auteur qu'il est devenu notamment en raison de certaines caractéristiques que l'on pourrait qualifier de « roumaines ", mais il nous semble tout aussi indiscutable que c'est surtout le choix d'écrire en français, depuis la France, pour les théâtres français, qui lui a permis de devenir un auteur mondial, qui parle à tous, et qui a contribué à former l'imaginaire de tous. 
Nous posons la question : Ionescu pouvait-il devenir un auteur canonique et mondial s'il avait continué à écrire, à publier et à mettre en scène ses pièces en roumain? Nous n'en sommes pas certains, nous en doutons même. Ou pour mieux le dire : nous pensons qu'on lui aurait attribué une reconnaissance internationale, mais que, quoi qu'il en soit, il n'aurait pas eu le même impact immédiat et « hégémonique » qu'il a eu, en tant qu'auteur français.

Et pourtant, cela peut arriver! Nous ne devons pas l'oublier. Dans le champ littéraire plus encore que dans le champ culturel, il est tout à fait possible que des zones les plus en retard ou les moins développées du monde proviennent des œuvres susceptibles d'impressionner et aussi de modifier l'imaginaire collectif mondial. Par une série de combinaisons, bien sûr, mais aussi en raison de la valeur intrinsèque du livre. Nous insistons sur l'adjectif " intrinsèque " parce que nous ne croyons pas que les interprétations sociologiques à la Bourdieu puissent expliquer à elles seules l'importance internationale d'auteurs comme Ionescu, Cioran, Eliade, comme semble le croire Mihai Iovănel, lequel analyse le succès à l'étranger de ces trois auteurs en termes de stratégies de marché ou en tout cas de lutte pour obtenir des positions de prestige dans le champ littéraire français ${ }^{21}$.

Ce que tente Iovănel est une opération typique de déconstruction du prestige des écrivains qui méconnaît, selon nous, la force cognitive ou poétique de leurs œuvres et semble par conséquent inspirée par la rancœur envers la créativité littéraire, comme aimait le dire Harold Bloom : aucune stratégie de marché (de rebrending, comme le dit Iovănel) ne peut expliquer pourquoi les publics continuent de rire de bon cœur depuis soixante-dix ans lors des représentations de La cantatrice chauve; de même qu'aucune considération sociologique ne peut expliquer pourquoi un roman comme Le Guépard, écrit par un auteur dilettante né dans une périphérie du monde et qui porte sur une réalité on ne peut plus provinciale, comme la Sicile entre le dix-neuvième et le vingtième siècle, est devenu un classique du $\mathrm{XX}^{\mathrm{e}}$ siècle. Et le cas du Guépard nous permet d'éclaircir deux autres nœuds à la fois critiques et littéraires qui ont aussi été abordés par les auteurs de ce livre ${ }^{22}$.

Le premier : ce ne sont pas toujours les Roumains ou les Italiens qui lisent, aiment, comprennent les livres écrits par les auteurs roumains ou italiens ; parfois, ce sont les autres, les lecteurs étrangers, comme si le potentiel sémantique et esthétique de certaines œuvres d'art pouvait être mieux capté si l'on se place depuis une perspective extérieure. Et de façon plus générale nous pourrions dire que l'on comprend mieux les classiques de la littérature de son propre pays si on devient, dans les limites du possible, étrangers à ceux-ci, c'est-à-dire si on les lit en adoptant une perspective de mise à distance. C'est précisément ce que, d'après nous, ne font pas nombre d'italianistes, mais peut-être aussi de spécialistes de la littérature roumaine, choisissant de lire les textes à l'intérieur de ces boîtes fermées et asphyxiantes que sont les histoires nationales de la littérature.

Mais c'est la seconde raison pour laquelle nous avons mentionné Le Guépard qui nous intéresse davantage encore. $\mathrm{Si}$ les distant readings à la Franco Moretti ne nous convainquent jamais complètement, c'est notamment parce qu'elles mettent en exergue les éléments quantitatifs par rapport aux éléments qualitatifs. La perspective darwinienne et statistique privilégie, en effet, la quantité sur la qualité, estimant que 
certains changements décisifs de style et de goût s'expliquent sur la base de grandes tendances historiques et sociales, pour ne pas dire de modes, tandis que, d'après nous, bien qu'il soit vrai que les œuvres littéraires expriment les désirs et les peurs profondes d'une époque, il n'en demeure pas moins vrai que ce sont les grands écrivains qui le font le mieux, et que même lorsque ceux-ci ne vendent pas beaucoup, ils influencent de façon indélébile l'imaginaire littéraire.

Il suffit de penser à Kafka. Il appartenait à la communauté juive et sa nationalité tchèque était plus que jamais périphérique, il a peu publié de son vivant et il est mort presque inconnu. De plus, l'image du monde qu'il proposait était tout sauf rassurante, et ne supportait évidemment aucune idéologie dominante. Et pourtant... nous ne croyons pas qu'il soit un auteur qui ait influencé plus que lui notre vision du monde. Aucune distant reading, aucune approche statistique et numérique ne pourront jamais en rendre compte.

Et cela ne vaut pas seulement pour des auteurs spécifiques, mais aussi pour des mouvements et des groupes d'écrivains. Prenons les romanciers russes du $\mathrm{XIX}^{\mathrm{e}}$ siècle, et hispano-américains des années 1950, 1960 et 1970 : ils ont révolutionné le roman occidental bien qu'appartenant aux périphéries ou semi-périphéries du système-monde. Mais dans un cas comme dans l'autre, il s'agit de réponses, de réactions aux initiatives prises auparavant par d'autres, c'est-à-dire d'élaborations originales d'un genre discursif (celui du roman moderne né entre la France et l'Angleterre) qui est parvenu à ces auteurs des métropoles et qu'ils ont su adapter à leur réalité.

Et cela signifie presque toujours que ces romans thématisent le retard de leur nation en nous racontant l'impact déconcertant que les produits économiques et culturels occidentaux ont eu sur les cultures périphériques, qu'elles soient russes, latino-américaines, japonaises ou africaines. Que l'on pense ici à Dostoïevski qui ne fait pas autre chose que se mesurer aux effets que l'individualisme et l'athéisme ont eus sur la société russe encore empreinte de christianisme et de féodalisme.

Il nous semble que c'est précisément sous cet aspect que le sens très aigu de l'absurde qui caractérise l'œuvre de Tristan Tzara, le prophète du dadaïsme, mais surtout des deux génies tardifs que sont Ionescu et Cioran, peut être interprété : seul qui était né dans un monde dans lequel tout avait encore un ordre et un sens métaphysique pouvait percevoir avec tant d'intensité et, pourrions-nous dire, de scandale, la perte de ces sécurités, pour dénoncer, ensuite, avec rage ou pour s'en moquer, la condition humaine comme insensée. Nous nous trouvons toujours du côté de ce phénomène que nous avons décrit comme le privilège des périphéries. Et pour bien s'entendre, il suffit d'esquisser une comparaison avec Sartre qui avait des liens beaucoup plus raisonnables et cordiaux avec le néant et l'absurde.

Mais nous pourrions dire que dans l'ensemble tout le modernisme roumain tel qu'il se développa entre les deux guerres se place sous le signe de l'anti-modernité et donc de l'expression d'un malaise profond devant des phénoménologies telles que la démocratie de masse, l'individualisme, le désenchantement religieux, le caractère envahissant de la technique ${ }^{23}$. Cela a été une sorte de grand laboratoire d'idées dans lequel la perspective révolutionnaire et la perspective conservatrice se sont souvent 
alternées mais aussi superposées, comme nous l'explique efficacement Paul Cernat :

Ce que nous devons aussi garder à l'esprit, c'est que les relations entre l'avant-garde et la jeune génération, et plus largement entre l'extrême droite et l'extrême gauche, ont été caractérisées par des chevauchements déconcertants, des similitudes, des associations, de même que par une circulation intense d'auteurs et d'idées dans les deux directions ${ }^{24}$.

Mais encore une fois, nous dirions que cela ne dépend pas d'une mauvaise élaboration des suggestions qui proviennent de l'Occident, mais cela s'explique en tenant compte du fait que dans la périphérie roumaine émerge une vérité malcommode : que les oppositions entre les positions culturelles extrêmes (politiques et poétiques) qui occupaient le devant de la scène entre les années vingt et les années quarante dans toute l'Europe celaient des affinités profondes.

Mais beaucoup des travaux adoptent cette double perspective : d'un côté, ils nous montrent comment la littérature roumaine du $\mathrm{XX}^{\mathrm{e}}$ siècle s'est servie de procédés et de modalités de représentation empruntés à des écrivains et des poètes centraux, mais d'un autre côté, ils nous montrent que, ce faisant, elle les démonte, les parodie, en pointe les limites, qui se font jour lorsqu'ils sont appliqués à des réalités périphériques.

On pense à l'usage original que certains poètes roumains, dans les années 1980 , ont fait de la poésie beat, et surtout de celle d'Allen Ginsberg : ils ont utilisé certaines formes de cette poésie selon des modalités que nous définirions, au sens large, d'ironiques car capables de faire ressortir, par contraste, les aspects arriérés, provinciaux de la réalité roumaine. Mais l'ironie concerne aussi ensuite la "manière beat " qui est démystifiée précisément à partir de l'incapacité de son style emphatique à rendre compte d'une réalité présumée provinciale, comme le démontre Teodora Dumitru ${ }^{25}$.

Tout cela pour dire que nous avons vraiment besoin d'un comparatisme qui, dans l'évaluation des phénomènes littéraires, ne nie pas que le monde fonctionne selon des rythmes et des vitesses différents, et prenne en considération la contemporanéité du non-contemporain, c'est-à-dire la coprésence de plusieurs temps historiques et donc de plusieurs mentalités au sein d'une même époque. Cela me paraît être la perspective le plus fructueuse pour une approche qui se veut radicalement comparatiste. On en trouve certains aperçus consistants dans cet ouvrage, mais qui mériteraient d'être développés davantage.

\section{BibLIOGRAPHIE}

Adamson, Walter L., Hegemony and Revolution : A Study of Antonio Gramscis Political and Cultural Theory, Berkeley, University of California Press, 1980.

Anderson, Benedict, Imagined Communities : Reflections on the Origin and Spread of Nationalism, Londres, New York, Verso, 1983.

Arac, Jonathan, «Angloglobalism? », in New Left Review 16, Juillet-Août, 2002, p. 35-45.

Brugnolo, Stefano, "The Pendulum Swing and the Privilege of Backwardness », in Paola Mildonian (dir.), It Started in Venice: Legacies, Passages, Horizons. Fifty Years of ICLA, Venise, Ca' Foscarina, 2009, p. 413-421. 
Casanova, Pascale, La république mondiale des lettres, Paris, Éditions du Seuil, 1999.

Compagnon, Antoine, Les antimodernes de Joseph de Maistre à Roland Barthes, Paris, Gallimard, 2005.

Martin, Mircea, Christian Moraru et Andrei Terian (dir.), Romanian Literature as World Literature, New York, Bloomsbury, 2018.

Moretti, Franco, « Conjectures on World Literature », in New Left Revieww, Nouvelle Série 1, janvier-février 2000.

Orlando, Francesco, L'intimité et l'bistoire. Une lecture du Guépard, Paris, Garnier, 2014.

Wallerstein, Immanuel, The Capitalist World-Economy, Cambridge, University Press, 1979.

\section{Notes}

1. Mircea Martin, Christian Moraru et Andrei Terian (dir.), Romanian Literature as World Literature, New York, Bloomsbury, 2018.

2. Christian Moraru and Andrei Terian, «Introduction: The Worlds of Romanian Literature and the Geopolitics of Reading», in Romanian Literature as World Literature, p. 1-32.

3. Ibid., p. 12-13. Le texte original, dont nous avons donné la traduction en français ici et dans tout l'article, est le suivant : « art cannot be explained as a social activity that fulfills the stated goals of a national [...] agenda ", et « the specific place of art is now increasingly located in networks that are both above and below the reach of the nation-state ».

4. Alex Goldiș, « Beyond Nation Building: Literary History as Transnational Geolocation», in Romanian Literature as World Literature, p. 95-113: 107. Voici de suite le fragment en original, qui est cité dans l'article dans notre traduction : " a country literature is a 'tangle of relations' that exceed by far national space, more exactly, the territory under that country's administration »; et encore : « there is nothing natural or selfevident about the concept of literary history as territorially organic construct, according to which literary and national borders are the same ».

5. Cfr. Immanuel Wallerstein, The Capitalist World-Economy, Cambridge, University Press, 1979.

6. Benedict Anderson, Imagined Communities: Reflections on the Origin and Spread of Nationalism, Londres, New York, Verso, 1983.

7. Christian Moraru et Andrei Terian, «Introduction», in Romanian Literature as World Literature, p. 18. Voici de suite le fragment en original : « this space is not set up according to a hierarchy of centers and margins. Nor does it look anything like the territories of those countries that put up walls and fences to keep immigrants, refugees, and other undesirables out ».

8. Ibid., p. 5. Voici de suite le texte en original : « aesthetically inferior reproduction of the core - hence the kindred complexes of 'marginality', 'provinciality', 'backwardness', 'derivativeness', 'belatedness', and 'inferiority' ".

9. Ibid., p. 15-16. Voici de suite le fragment en original : « cultural, and epistemological scalarity has been the nation-state, through which the human, in its cultural expression, and the human world become intelligible to humanists for almost 200 years ». Et encore : "It urges on us the entire planet as a unity of analysis ».

10. Ibid.,p. 19. Voici de suite le fragment en original : «What literature is not nomadic? What literature is not 'immigrant'? What literature does not depend on - and sometimes is even 'born' - in translation? [...] And what literature production is not transcultural and transterritorial? ».

11. Cfr. Franco Moretti, "Conjectures on World Literature ", in New Left Review, Nouvelle Série 1, janvier-février 2000.

12. Christian Moraru et Andrei Terian, «Introduction», in Romanian Literature as World Literature, p. 4. Voici de suite le fragment en original : « treating national literature as a 'particular nodal point'(Beebee) in a larger unit ». Et encore : " rather simplistic view of cultural mechanics regulating centers and margins ». 13. Ibid., p. 17. Voici de suite le fragment en original : " a colorful and pulsing cross-section of everything meeting, touching, classifying, mixing and passing in it and through it [la nation]». 
14. Ibid., p.14. Voici de suite le texte original : « literature for the planet is 'an off-center set of vibrations, chaotic and tangential', a field of energies, a flow ».

15. Cfr. Pascale Casanova, La république mondiale des lettres, Paris, Éditions du Seuil, 1999.

16. Teodora Dumitru, "Gaming the World-System: Creativity, Politics, and Beat Influence in the Poetry of the 1980s Generation ", in Romanian Literature as World Literature, p. 284. Voici de suite le texte original : « exists within a constant flux of translations from other world texts and for the world market ».

17. On peut lire à ce sujet ce qu'écrit Jonathan Arac dans "Angloglobalism ? ", Nerw Left Revierw 16, Juillet-Août, $2002: 35-45$, p. 38. La traduction nous appartient : " La mondialisation pluralise : elle ouvre toutes les cultures locales, nationales ou régionales aux autres et produit par là même 'de nombreux mondes'. Et pourtant ces nombreux mondes ne peuvent être connus qu'à travers un seul moyen : de même que le dollar est le moyen du commerce global, l'anglais est le moyen de la culture globale, qui produit 'un seul monde'».

18. Cfr. Walter L. Adamson, Hegemony and Revolution: A Study of Antonio Gramsci's Political and Cultural Theory, Berkeley, University of California Press, 1980.

19. Stefano Brugnolo, "The Pendulum Swing and the Privilege of Backwardness ", in Paola Mildonian (dir.), It Started in Venice: Legacies, Passages, Horizons. Fifty Years of ICLA, Venise, Ca' Foscarina, 2009, p. 420: «We can speak of deferred or delayed answers from the Periphery as response to the processes of modernisation initiated by the Centre. It is almost as if the peripheral writers possessed, at least on a theoretical level, the privilege of later reconsidering and redefining those processes that elsewhere had become stabilised, a sort of second nature. I have termed this phenomenon the privilege of backwardness ».

20. Christian Moraru et Andrei Terian, «Introduction», in Romanian Literature as World Literature, p. 20. Voici de suite le fragment en original : "One core tenet of the essays in Romanian Literature as World Literature holds that $[\ldots]$ this literature has come about by asserting its planetary belonging, its being in the world, for it, and of it ".

21. Cfr. Mihai Iovănel, «Temporal Webs of World Literature: Rebranding Games and Global Relevance after the Second World War - Mircea Eliade, E. M. Cioran, Eugène Ionesco », in Romanian Literature as World Literature, p. 217-234.

22. Cfr. Francesco Orlando, L'intimité et l'bistoire. Une lecture du Guépard, Paris, Garnier, 2014.

23. Cfr. Antoine Compagnon, Les antimodernes de Joseph de Maistre à Roland Barthes, Paris, Gallimard, 2005.

24. Cfr. Paul Cernat, «Communicating Vessels : The Avant-Garde, Antimodernity, and Radical Culture in Romania between the First and the Second World Wars ", in Romanian Literature as World Literature, p. 195-214: 198. Voici de suite le fragment en original : "What we should also keep in mind is that the relationships between the Young Generation, and more broadly between the Far Right and the Far Left were characterized by baffling overlaps, similitudes, associations, as well as an intense circulation of authors and ideas in both directions ".

25. Cfr. Teodora Dumitru, « Gaming the World-System : Creativity, Politics, And Beat Influence in the Poetry of the 1980s Generation », p. 271-287: 274-278. 